\title{
A FEW MORE BALANCED ROOM SQUARES
}

\author{
D. R. STINSON and S. A. VANSTONE
}

(Received 2 May 1984)

Communicated by W. D. Wallis

\begin{abstract}
The existence problem for balanced Room squares is, in general, unsolved. Recently, B. A. Anderson gave a construction for a class of these designs with side $2^{n}-1$, where $n$ is odd and $n \geqslant 3$. For $n$ even, the existence has not yet been settled. In this paper, we use the affine geometry of dimension $2 k$ and order 2, and a hill-climbing algorithm, to construct a number of new balanced Room squares directly. Recursive techniques based on finite geometries then give new squares of side $2^{2 k}-1$ for infinitely many values of $k$.
\end{abstract}

1980 Mathematics subject classification (Amer. Math. Soc.): 05 B 25.

\section{Introduction}

A Room square of side $r$ defined on an $(r+1)$-set $V$ is an $r \times r$ array $A$ satisfying the following conditions:

(1) each cell of $A$ is either empty or contains an unordered pair of distinct elements from $V$;

(2) each element of $V$ is contained in precisely one cell of each row and each column of $A$;

(3) every pair of distinct elements from $V$ is contained in exactly one cell of $A$. We denote a Room square of side $r$ by $R S(r)$.

The spectrum for Room squares was determined in 1973.

THEOREM 1.1 (Mullin and Wallis [8]). There exists an $R S(r)$ if and only if $r$ is an odd positive integer other than 3 or 5 .

(C) 1985 Australian Mathematical Society $0263-6115 / 85 \$ A 2.00+0.00$ 
A Room square $R S(r)$ defined on set $V$ is said to be ordered if every pair $\{x, y\}$ in the array is replaced by one of the ordered pairs $(x, y)$ or $(y, x)$. Let $F_{i}$ denote the set of all first components taken from the ordered pairs in row $i$ and let $S_{i}$ denote the set of all second components in row $i$. Clearly, $F_{i} \cup S_{i}=V$, $1 \leqslant i \leqslant r$. Let $\beta=\left\{F_{i}, S_{i}: 1 \leqslant i \leqslant r\right\}$.

If $(V, \beta)$ is a balanced incomplete block design, then the ordered Room square is called a balanced Room square of side $r$ and is denoted $B R S(r)$.

\section{EXAMPLE}

\begin{tabular}{|r|r|r|r|r|r|r|}
\hline $0 \infty$ & 26 & 45 & & 13 & & \\
\hline & $1 \infty$ & 30 & 56 & & 24 & \\
& & $2 \infty$ & 41 & 60 & & 35 \\
46 & & & $3 \infty$ & 52 & 01 & \\
& 50 & & & $4 \infty$ & 63 & 12 \\
23 & & 61 & & & $5 \infty$ & 04 \\
\hline 15 & 34 & & 02 & & & $6 \infty$ \\
\hline
\end{tabular}

$$
\begin{array}{ll}
F_{1}=\{0,2,4,1\}, & S_{1}=\{\infty, 5,5,3\} . \\
F_{2}=\{1,3,5,2\}, & S_{2}=\{\infty, 0,6,4\} . \\
F_{3}=\{2,4,6,3\}, & S_{3}=\{\infty, 1,0,5\} . \\
F_{4}=\{4,3,5,0\}, & S_{4}=\{6, \infty, 2,1\} . \\
F_{5}=\{5,4,6,1\}, & S_{5}=\{0, \infty, 3,2\} . \\
F_{6}=\{2,6,5,0\}, & S_{6}=\{3,1, \infty, 4\} . \\
F_{7}=\{1,3,0,6\}, & S_{7}=\{5,4,2, \infty\} .
\end{array}
$$

$\mathrm{A} B R S(7)$

A balanced Room square $B R S(r)$ is sometimes called a complete balanced Howell rotation on $(r+1)$ teams. This terminology arises from some of the early history of the subject in connection with designing duplicate bridge tournaments with various properties. The interested reader is referred to [9].

Unlike Room squares, the existence question for balanced Room squares is unsolved. It is well known $[3,9,10]$ that a necessary condition for a $B R S(r)$ to exist is $r \equiv 3(\bmod 4)$. In this paper, we are interested in the existence of $B R S\left(2^{n}-1\right)$. In a recent paper by B. Anderson [1], it is shown that for all odd $n \geqslant 3$, such a design exists. We will briefly consider this construction in the next section. In Section 3 we construct by direct methods a number of new $B R S\left(2^{n}-1\right)$ for various even values of $n$ and in Section 4 recursive techniques are applied to produce infinitely many new squares. The constructions considered throughout this paper are based on finite affine geometries.

We identify the points of $A G(n, 2)$, the affine geometry of order 2 and dimension $n$, with the elements of the field $G F\left(2^{n}\right)$. Let $\alpha$ be a generator for $G F\left(2^{n}\right)$ and define $\alpha^{\infty}=0$. Lines in this geometry contain two points. If $L=\left\{\alpha^{a}, \alpha^{b}\right\}$ is a line, then we will usually write this as $L=\{a, b\}$. Two lines $L_{1}=\left\{\alpha^{a}, \alpha^{b}\right\}$ and $L_{2}=\left\{\alpha^{c}, \alpha^{d}\right\}$ are parallel if and only if $\alpha^{a}+\alpha^{b}=\alpha^{c}+\alpha^{d}$. A set of lines $S=\left\{L_{i}: 1 \leqslant i \leqslant 2^{n-1}\right\}$ is said to be a skew resolution class of the 
geometry if $S$ is a partition of the point set and no two distinct lines of $S$ are parallel. A skew resolution is a set of skew resolution classes which partition the lines of $A G(n, 2)$. We will make extensive use of the following result.

THEOREM 1.2 (Fuji-Hara and Vanstone [7]). If there exists a skew resolution in $A G(n, 2)$, then there exists a BRS $\left(2^{n}-1\right)$.

ProOf. Let $r=2^{n}-1$ and $P_{1}, P_{2}, \ldots, P_{r}$ be the $r$ parallel classes of lines in the geometry. Let $R_{1}, R_{2}, \ldots, R_{r}$ be $r$ skew resolution classes forming a skew resolution. Index the rows and columns of an $r \times r$ array $A$ by the parallel classes and skew resolution classes respectively. In cell $\left(P_{i}, R_{j}\right)$ place the set $P_{i} \cap R_{j}$. Clearly, every cell is either empty or contains a line of $A G(n, 2)$. It is easy to see that $A$ is a Room square. We now order the pairs in $A$ to form a balanced Room square.

Since $P_{i}$ is a parallel class of lines there is a unique pair of parallel hyperplanes $F_{i}$ and $S_{i}$ such that each line of $P_{i}$ contains one point from each. If $L=\{x, y\} \in P_{i}$ and $x \in F_{i}, y \in S_{i}$, then replace $L$ in $A$ by $(x, y)$. Since the set of all hyperplanes forms a $B I B D$ the resulting array is a $B R S\left(2^{n}-1\right)$.

\section{Preliminaries}

In [1], B. A. Anderson proved the existence of a $B R S\left(2^{n}-1\right)$ for all odd $n \geqslant 3$. In this section, we briefly describe this construction in terms of affine geometries and Theorem 1.2 above.

Consider $A G(n, 2)$ ( $n$ odd, $n \geqslant 3$ ) and let $\alpha$ be a generator for $G F\left(2^{n}\right)$. Let

$$
P=\left\{\left\{\alpha^{i}, 1+\alpha^{i}\right\}: i=\infty, 0 \leqslant i \leqslant 2^{n}-2\right\} .
$$

Then $P$ is a parallel class of lines which generates all other parallel classes of lines under a cyclic automorphism of order $2^{n}-1$. Let

$$
S=\left\{\left\{\alpha^{\infty}, \alpha^{0}\right\}\right\} \cup\left\{\left\{1 / \alpha^{i}, 1 /\left(1+\alpha^{i}\right)\right\}: 0 \leqslant i \leqslant 2^{n}-2\right\} .
$$

Then $S$ is a skew resolution class of lines which generates a skew resolution under the action of the automorphism group of order $2^{n}-1$. It is not difficult to show that $S$ is a partition of the point set. Suppose two lines of $S$ are parallel. For some $i$ and $j$ then either

(I) $1 / \alpha^{i}+1 /\left(1+\alpha^{i}\right)=1 / \alpha^{j}+1 /\left(1+\alpha^{j}\right)$ or

(II) $\alpha^{\infty}+\alpha^{0}=1 / \alpha^{i}+1 /\left(1+\alpha^{i}\right)$.

In (I) we get that $\left(\alpha^{i}+\alpha^{j}\right)+\left(\alpha^{i}+\alpha^{j}\right)=0$ implying $\alpha^{i}+\alpha^{j}=0$ or $\alpha^{i}+\alpha^{j}=1$. In either case the two lines are identical. In (II), $1=1 / \alpha^{i}\left(1+\alpha^{i}\right)$ or $\alpha^{2 i}+\alpha^{i}+1$ $=0$. Hence $\alpha^{i}$ is a cube root of unity (not 1 ), which is impossible since $n$ odd implies $2^{n}-1$ is not divisible by 3 . We summarize this in the following theorem. 
THEOREM 2.1. There exists a cyclically generated skew resolution in $A G(n, 2)$ for all odd $n \geqslant 3$.

COROLLARY 2.1. There exists a BRS $\left(2^{n}-1\right)$ for all odd $n \geqslant 3$.

It is easy to see why the construction given here fails for $n$ even. The lines $\{0, \infty\}$ and $\left\{\left(2^{n}-1\right) / 3,\left(2^{n+1}-2\right) / 3\right\}$ are both in $P$ and $S$ and, hence, $S$ is not a skew resolution class.

We require several more definitions. The concepts of starter adder and strong starter are fundamental in the study of Room squares.

A starter $T$ in a finite abelian group $G$ of odd order is a partition of $G \backslash\{0\}$ into pairs which form a difference set (i.e., $\{ \pm(a-b):\{a, b\} \in T\}=G \backslash\{0\}$ ).

An adder $A$ for a starter $T$ in $G$ is an injective mapping from $T$ into $G \backslash\{0\}$ such that

$$
\{a+A(\{a, b\}), b+A(\{a, b\}):\{a, b\} \in T\}=G \backslash\{0\} .
$$

If $T$ is a starter and $\Sigma T=\{a+b:\{a, b\} \in T\}$ is a set of $|T|$ distinct elements of $G \backslash\{0\}$ then $T$ is called a strong starter.

It is well known [8] that a starter and adder in a finite abelian group $G$ of order $r$ imply the existence of an $R S(r)$ and that a strong starter in $G$ implies the existence of a starter and adder in $G$.

Consider the parallel class $P$ in $A G(n, 2)$ as given above. $P$ is a parallel class for all values of $n$. If $\left\{\alpha^{i}, 1+\alpha^{i}\right\}$ is a line of $P$, then replace this pair by $\{i, z(i)\}$ where $1+\alpha^{i}=\alpha^{z(i)}$ to get a set of pairs $P^{\prime} ; P^{\prime}$ is a starter in $G=Z_{r}$ where $r=2^{n}-1$. Anderson showed that $P^{\prime}$ is a strong starter in $G$. This is, of course, not true when $n$ is even. In the next section we construct adders for the starter $P^{\prime}$ for various values of $n$ even. It should be clear that $P^{\prime}$ and an adder will produce a skew resolution class which will generate a skew resolution.

\section{Some new balanced room squares}

Given a starter in a finite abelian group $G$, Dinitz and Stinson [4] have devised a hill-climbing algorithm for finding an adder for the starter. Applying this algorithm to $P^{\prime}$ of the previous section we obtain the following results.

THEOREM 3.1. There exists a skew resolution in $A G(n, 2)$ for $n=4,6,8$ and 14 .

Proof. For $n=4,6,8$, we list the polynomial used to define $G F\left(2^{n}\right)$, the parallel resolution class $P^{\prime}$, an adder $A$ for $P^{\prime}$, and the corresponding skew resolution class $S$. 


\begin{tabular}{|c|c|c|}
\hline$P^{\prime}$ & $A$ & $S$ \\
\hline 11,12 & 1 & 12,13 \\
\hline 7,9 & 14 & 6,8 \\
\hline 1,4 & 3 & 4,7 \\
\hline 14,3 & 2 & 1,5 \\
\hline 5,10 & 4 & 9,14 \\
\hline 2,8 & 9 & 11,2 \\
\hline 6,13 & 12 & 3,10 \\
\hline
\end{tabular}

$n=6 \quad f(x)=x^{6}+x+1$

\begin{tabular}{|c|c|c|c|c|c|c|c|c|c|c|c|}
\hline$P^{\prime}$ & $A$ & $S$ & $P^{\prime}$ & $A$ & $S$ & $P^{\prime}$ & $A$ & $S$ & $P^{\prime}$ & $A$ & $S$ \\
\hline 57,58 & 59 & 53,54 & 18,27 & 25 & 43,52 & 16,33 & 13 & 29,46 & 52,14 & 7 & 59,21 \\
\hline 51,53 & 26 & 14,16 & 2,12 & 6 & 8,18 & 36,54 & 58 & 31,49 & 56,19 & 5 & 61,24 \\
\hline 31,34 & 39 & 7,10 & 38,40 & 37 & 12,23 & 7,26 & 43 & 50,6 & 45,9 & 38 & 20,47 \\
\hline 39,43 & 23 & 62,3 & 61,10 & 41 & 39,51 & 4,24 & 36 & 40,60 & 22,50 & 46 & 5,33 \\
\hline 1,6 & 35 & 36,41 & 28,41 & 4 & 32,45 & 21,42 & 34 & 55,13 & 3,32 & 53 & 56,22 \\
\hline 62,5 & 12 & 11,17 & 11,25 & 33 & 44,58 & 13,35 & 2 & 15,37 & 17,47 & 17 & 34,1 \\
\hline 37,44 & 28 & 2,9 & 40,55 & 50 & 27,42 & 48,8 & 40 & 25,48 & 29,60 & 60 & 26,57 \\
\hline 15,23 & 15 & 30,38 & 30,46 & 52 & 19,35 & 59,20 & 8 & 4,28 & & & \\
\hline
\end{tabular}




$$
n=8 \quad f(x)=x^{8}+x^{4}+x^{3}+x^{2}+1
$$

\begin{tabular}{|c|c|c|c|c|c|c|c|c|c|c|c|}
\hline$P^{\prime}$ & $A$ & $S$ & $P^{\prime}$ & $A$ & $S$ & $P^{\prime}$ & $A$ & $S$ & $P^{\prime}$ & $A$ & $S$ \\
\hline & 41 & & & 2 & & & 68 & & 59 & 129 & 29,3 \\
\hline & 94 & & & 86 & & & & & & 0 & \\
\hline 13 & 05 & 40 , & 4,244 & 82 & 1 & & 215 & & & 242 & \\
\hline & 53 & & & 143 & 174,1 & 22 & 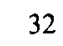 & & & 224 & \\
\hline & 55 & & & 79 & & & & & 213,2 & t & \\
\hline & 6 & & & 30 & & & 99 & & & 197 & 222 \\
\hline 25 & 140 & 1 & 83 & 217 & 5 & 78 & 15 & & & 113 & \\
\hline 74 & 96 & 9 & 19 & 122 & 56 & 1 & 6 & & 220 & 189 & \\
\hline 240 & 25 & & & r & & & & & & 170 & \\
\hline 76,11 & 188 & & & 37 & 16 & 149 & 205 & & 171,211 & 132 & 48,88 \\
\hline & 199 & & 2 & 52 & & & 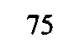 & & & 107 & 147,191 \\
\hline & 164 & & & 65 & & & & & & 80 & \\
\hline 58 & 1 & & & 167 & 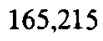 & & 2 & & 114 & 29 & \\
\hline & I & & & 212 & & & & & & 124 & \\
\hline 172,2 & 1 & & 16 & 216 & & & 1 & & & 8 & \\
\hline 69,13 & 100 & 169 & 165,227 & 62 & 227 & & 120 & & 185 & 206 & 136,200 \\
\hline & 198 & & & & & & 133 & & & & \\
\hline 125,19 & 3 & & 191,6 & 213 & 149 & & 78 & & & 247 & 52,124 \\
\hline 19,9 & 218 & & 152,226 & 92 & 224 & & 22 & & 142 & 115 & 78 \\
\hline & & & & 4 & & & & & & & \\
\hline & 16 & & 196,23 & 150 & 91 & 198 & 178 & 04 & 215,44 & 222 & 182,11 \\
\hline & & & & & & & & & & 11 & \\
\hline & & & & 173 & & & 18 & & & 170 & \\
\hline 97 & 181 & & 47 & 84 & 137 & & 7 & & 0 & 110 & 114 \\
\hline & & & 200 & 23 & & & 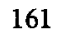 & & & 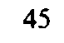 & \\
\hline 208,54 & 169 & & & & & & 33 & 62 & 228,77 & 184 & 157,6 \\
\hline & 59 & & & 223 & 184 & 197 & 0 & & & 40 & \\
\hline & 69 & & 129,239 & 134 & & & 108 & & 248,105 & 136 & 129,241 \\
\hline & 56 & & & 204 & & & & & 41 , & 61 & 102,218 \\
\hline & 73 & & & 201 & & 102 & 165 & & 246,111 & 24 & \\
\hline 212,78 & 156 & 113,234 & 138,5 & 39 & 177,44 & & 51 & 123,246 & 75,199 & 145 & 220,89 \\
\hline 61,186 & 128 & & 145,16 & 35 & 180,51 & 243,115 & 93 & 81,208 & & & \\
\hline
\end{tabular}

In $A G(14,2)$ there are 16,384 points and a skew resolution contains 8,192 lines. We omit a listing of the skew resolution found. The hill-climbing algorithm took 11 seconds of CUP time on an Amdahl 580 computer to find an adder for the parallel class $P^{\prime}$.

COROLlary 3.1. There exists a BRS $\left(2^{n}-1\right)$ for $n=4,6,8$ and 14 . 
The case $n=6$ was recently done independently by B. A. Anderson [2] using a related but somewhat different approach. Anderson begins with a pair of parallel hyperplanes in $A G(6,2)$ which he writes as a pair of supplementary difference sets $A$ and $B$ in $Z_{63}$.

$$
\begin{array}{r}
A=\{\infty, 1,2,3,4,5,7,8,9,10,13,14,15,17,19,20,25,27,28,29,33,34,36,37, \\
39,42,46,49,50,53,55,57\} . \\
B=\{0,6,11,12,16,18,21,22,23,24,26,30,31,32,35,38,40,41,43,44,45,47, \\
48,51,52,54,56,58,59,60,61,62\} .
\end{array}
$$

Using a modified version of the Dinitz-Stinson hill-climbing algorithm he constructs a strong starter $T$ in $Z_{63}$ where each pair in $T$ contains one element from $A$ and one from $B$. The strong starter $T$ is listed below.

$$
\begin{aligned}
& \{46,45\},\{33,35\},\{9,6\},\{57,61\},\{53,48\},\{49,43\},\{37,44\}, \\
& \{13,21\},\{7,16\},\{36,26\},\{20,31\},\{5,56\},\{1,51\},\{25,11\},\{8,23\}, \\
& \{15,62\},\{29,12\},\{42,24\},\{10,54\},\{27,47\},\{39,18\},\{17,58\},\{55,32\}, \\
& \{2,41\},\{34,59\},\{14,40\},\{3,30\},\{50,22\},\{4,38\},\{19,52\},\{28,60\} .
\end{aligned}
$$

$T$ generates a $B R S(63)$ whose associated $B I B D$ is isomorphic to the design consisting of the points and hyperplanes of $A G(6,2)$. This $B R S(63)$ is not isomorphic to the one displayed in Theorem 3.1. Neither the rows nor the columns form the parallel resolution of lines in $A G(6,2)$.

\section{Recursive constructions}

Unlike the Room square case, there are very few recursive constructions for balanced Room squares. In terms of finite geometries and skew resolutions, several recursive constructions do exist. We will use these techniques to produce infinitely many new balanced Room squares.

The definition of skew resolution class and skew resolution extend to $A G(n, q)$.

ThEOREM 4.1 (Fuji-Hara and Vanstone [6]). If there exists a skew resolution in $A G(m, q)$ and a skew resolution in $A G\left(n, q^{m}\right)$, then there exists a skew resolution in $A G(m n, q)$.

THEOREM 4.2 (Fuji-Hara and Vanstone [6]). If there exists a skew resolution in $A G(m+1, q)$ and a skew resolution in $A G\left(n, q^{m}\right)$, then there exists a skew resolution in $A G(m n+1, q)$.

In order to apply Theorems 4.1 and 4.2 we need to know something about the existence of skew resolutions in $A G(n, q)$ for $q>2$. 
Theorem 4.3 (Fuji-Hara and Vanstone [5]). There exists a skew resolution in $A G\left(2^{i}-1, q\right)$ for all $q$ and $i \geqslant 2$. In particular, there exists a skew resolution in $A G\left(2^{i}-1,2^{j}\right)$ for all $i \geqslant 2, j \geqslant 2$.

Applying Theorems 4.1 and 4.3 we get the following theorem.

THEOREM 4.4. There exists a skew resolution in $A G\left(n\left(2^{i}-1\right), 2\right)$ for $n=4,6,8$ and 14 and all $i \geqslant 2$.

Applying Theorems 4.2 and 4.3 we get

THEOREM 4.5. There exists a skew resolution in $A G\left((n-1)\left(2^{i}-1\right)+1,2\right)$ for $n=4,6,8$ and 14 and all $i \geqslant 2$.

In terms of balanced Room squares we summarize the above results.

THEOREM 4.6. There exists a BRS( $r)$ for all

(1) $r=n\left(2^{i}-1\right)-1, i \geqslant 2, n=4,6,8$ and 14 ,

(2) $r=(n-1)\left(2^{i}-1\right), i \geqslant 2, n=4,6,8$ and 14 .

The smallest value of $k$ for which a skew resolution in $A G(2 k, 2)$ is not yet known to exist is $k=10$ and, in terms, of balanced Room squares the smallest $B R S\left(2^{2 k}-1\right)$ unknown is $k=10$.

\section{Conclusion}

In this paper we have been concerned with balanced Room squares of side $2^{n}-1$ where $n$ is even and we have constructed infinitely many new ones in this class. Since $B R S\left(2^{m}-1\right), m$ odd and $m \geqslant 3$, are all known to exist, what would be ideal is a recursive construction which doubles the point set. Such a construction would prove the existence of $B R S\left(2^{2 k}-1\right)$. Unfortunately, no such method is known. In terms of affine geometries the problem could be solved by showing that a skew resolution in a space of dimension $2^{k}-1$ implies the existence of a skew resolution in a space of dimension $2 k$. Again, such a construction is not known.

We finish by mentioning that it has recently been shown [11] that there are at least 279 non-isomorphic skew resolutions in $A G(5,2)$, and hence at least 279 non-isomorphic $B R S(31)$. 


\section{Acknowledgment}

The authors would like to thank J. H. Dinitz for his many helpful comments.

\section{References}

[1] B. A. Anderson, 'Hyperplanes and balanced Howell rotations,' Ars Combinatoria 13 (1982), 163-168.

[2] B. A. Anderson, private communication.

[3] E. R. Berlekamp and F. K. Hwang, 'Construction for balanced Howell rotations for bridge tournaments,' J. Combin. Theory 12 (1972), 159-166.

[4] J. H. Dinitz and D. R. Stinson, 'A fast algorithm for finding strong starters,' SIAM J. Algebraic Discrete Methods 2 (1981), 50-56.

[5] R. Fuji-Hara and S. A. Vanstone, 'Affine geometries obtained from projective planes and skew resolutions of $A G(3, q)$ ', Ann. Discrete Math. 18 (1983), 355-376.

[6] R. Fuji-Hara and S. A. Vanstone, 'Recursive constructions for skew resolutions in affine geometries', Aequationes Math. 23 (1981), 242-251.

[7] R. Fuji-Hara and S. A. Vanstone, 'Some results on balanced Room squares and their generalizations', preprint.

[8] R. C. Mullin and W. D. Wallis, 'The existence of Room squares', Aequationes Math. 13 (1975), $1-7$.

[9] E. T. Parker and A. M. Mood, 'Some balanced Howell rotations for duplication bridge sessions', Amer. Math. Monthly 26 (1955), 714-716.

[10] P. J. Schellenberg, 'On balanced Room squares and complete balanced Howell rotations', Aequationes Math. 9 (1973), 75-90.

[11] D. R. Stinson and S. A. Vanstone, 'Orthogonal packings in $P G(5,2)$ ', Geom. Dedicata, submitted.

Department of Computer

Science

University of Manibota

Winnipeg, Manitoba R3T 2N2

Canada
Department of Combinatorics and Optimization St. Jerome's College University of Waterloo Waterloo, Ontario N2L 3G1

Canada 